\title{
MEASUREMENT OF LINEAR COUPLING RESONANCE IN RHIC *
}

\author{
M. Bai, F. Pilat, T. Satogata \\ Brookhaven National Lab., Upton, NY 11973, USA \\ R. Tomas, F. Schmidt \\ CERN, CH-1211 Geneva 23. Switzerland \\ R. Calaga \\ State University of New York, StonyBrook, NY 11794, USA
}

\begin{abstract}
Linear coupling is one of the factors that determine beam lifetime in RHIC. The traditional method of measuring the minimum tune separation requires a tune scan and can't be done parasitically or during the acceleration ramp. A new technique of using ac dipoles to measure linear coupling resonance has been developed at RHIC. This method measures the degree of coupling by comparing the amplitude of the horizontal coherent excitation with the amplitude of the vertical coherent excitation if the beam is excited by the vertical AC dipole and vice versa. One advantage of this method is that it can be done without changing tunes from the normal machine working points. In principle, this method can also localize the coupling source by mapping out the coupling driving terms throughout the ring. This is very useful for local decoupling the interaction regions in RHIC. A beam experiment of measuring linear coupling has been performed in RHIC during its 2003 run, and the analysis of the experimental data is discussed in this paper.
\end{abstract}

\section{INTRODUCTION}

In a circular accelerator, the linear $4 \mathrm{x} 4$ one-turn transfer matrix $T$ for the two-dimensional phase space (x,x',y,y') can be represented by four $2 \times 2$ sub-matrices $M, m, n$ and N

$$
T=\left(\begin{array}{cc}
M & m \\
n & N
\end{array}\right)
$$

where $M$ and $N$ are the standard transfer matrices in the $\left(\mathrm{x}, \mathrm{x}^{\prime}\right)$ phase and $\left(\mathrm{y}, \mathrm{y}^{\prime}\right)$ phase space. The off-diagonal submatrices $m$ and $n$ represent the coupling between the two transverse planes. In a perfect accelerator with no coupling, $m=n=0$.

In general, the one turn matrix $T$ can be diagonalized by an canonical transformation

$$
T=R U R^{-1}
$$

where $U$ is the one-turn transfer matrix for the two decoupled transverse phase spaces (u,u',v,v') [1] and

$$
\left(\begin{array}{c}
x \\
x^{\prime} \\
y \\
y^{\prime}
\end{array}\right)=R\left(\begin{array}{c}
u \\
u^{\prime} \\
v \\
v^{\prime}
\end{array}\right) .
$$

\footnotetext{
* The work was performed under the auspices of the US Department of Energy
}

where $R$ is the transformation matrix between $(x, z, s)$ and $(u, v, s)$.

$$
R=\left(\begin{array}{cc}
\gamma I & C \\
-C^{+} & \gamma I
\end{array}\right)
$$

Here $I$ is the $2 \times 2$ unitary matrix and $C^{+}$is the simplectic conjugate matrix of the $2 \times 2$ matrix $C$

$$
C=\left(\begin{array}{ll}
c_{11} & c_{12} \\
c_{21} & c_{22}
\end{array}\right)
$$

and $\gamma^{2}+|C|=1$. When the $C$ matrix is zero, there is no coupling between the two transverse planes, and one can use $C$ to characterize the strength of the linear coupling.

A technique to measure $C$ by exciting beam at its eigentunes was developed at CESR [2]. Unlike CESR, an AC dipole was used in RHIC to adiabatically excite the beam at the vicinity of the eigen-tune instead. This technique has been used to measure the beta functions and phase advances in RHIC [4], and also in the AGS to overcome intrinsic spin resonances [3].

Without losing generality, we assume that the horizontal ac dipole (vertical field) is used to adiabatically excite a sizable coherent excitation nearby the horizontal tune $Q_{x}$. The horizontal AC dipole integrated field $\Delta B_{y} L$ is

$$
\Delta B_{y} L=\Delta B_{y m} L \cos \nu_{m} \theta
$$

where $\Delta B_{y m}$ is the amplitude of the oscillating field, $Q_{m}$ is the tune of the AC dipole field and $\theta$ is the azimuthal angle. In the absence of linear coupling, a horizontal betatron oscillation at location $s$ is excited with amplitude of

$$
x(s)=\frac{\Delta B_{y m} L}{4 \pi B \rho \delta_{x}} \sqrt{\beta_{0} \beta(s)}
$$

and no coherent excitation is excited in the vertical plane. Here, $B \rho$ is the magnetic rigidity, $\delta_{x}=\left|Q_{m}-Q_{x}\right|$ is the distance between the AC dipole driving tune $Q_{m}$ and horizontal betatron tune, $\beta_{0}$ is the beta-function at the $\mathrm{AC}$ dipole and $\beta_{s}$ is the $\beta$-function at location $s$. With linear coupling, the vertical coherent excitation is no longer zero. To derive the size of the coherent excitations in the two transverse planes due to an horizontal AC dipole, we first project the horizontal deflection from the AC dipole into the $(u, v)$ coordinate where the motions in the $\left(u, u^{\prime}\right)$ 
plane and $\left(v, v^{\prime}\right)$ are decoupled, i.e

$$
\left(\begin{array}{c}
\delta u \\
\delta u^{\prime} \\
\delta v \\
\delta v^{\prime}
\end{array}\right)=\left(\begin{array}{c}
0 \\
\gamma \delta x^{\prime} \\
-c_{12} \delta x^{\prime} \\
c_{11} \delta x^{\prime}
\end{array}\right) .
$$

where $\delta x^{\prime}$ is the deflection of the horizontal ac dipole. The coherent excitations in the $\left(u, u^{\prime}\right)$ and $\left(v, v^{\prime}\right)$ are given by

$$
\begin{array}{r}
u=\frac{1}{4 \pi \delta_{u}} \gamma \delta x^{\prime} \cos Q_{m} \theta \\
v=\frac{1}{4 \pi \delta_{v}} \sqrt{c_{12}^{2}+c_{11}^{2}} \delta x^{\prime} \cos Q_{m} \theta
\end{array}
$$

where $\delta_{v}=\left|Q_{m}-Q_{v}\right|$ and $\delta_{u}=\left|Q_{m}-Q_{u}\right| . Q_{v}$ and $Q_{u}$ are the two eigen-tunes of the transverse free betatron oscillation. For weak coupling,

$$
\begin{aligned}
Q_{u} & \simeq Q_{x} \\
Q_{v} & \simeq Q_{y} \\
\delta_{u} & \simeq \delta_{x} \\
\delta_{v} & \simeq \delta_{y} .
\end{aligned}
$$

In general, $\sqrt{c_{11}^{2}+c_{22}^{2}}$ is small for weak coupling and $\delta_{x}$ is much smaller than $\delta_{y}$ because the horizontal AC dipole drives the beam close to the horizontal tune. Hence, the coherent excitation in the $\left(v, v^{\prime}\right)$ space can be neglected. Transforming the driven coherent excitations in the $(u, v)$ coordinate back to the normal geometric $(x, y)$ coordinates using Eq. 3, the transverse coherent excitations at location $s$ are

$$
\begin{array}{r}
x(s)=\gamma \frac{\sqrt{\beta_{x}}}{4 \pi \delta_{x}}\left(\gamma \delta x^{\prime}\right) \cos \left(Q_{m} \theta+\chi\right) \\
y(s)=\frac{\sqrt{\beta_{y}}}{4 \pi \delta_{x}}\left(\gamma \delta x^{\prime}\right)\left(c_{22}^{-} \cos \left(Q_{m} \theta+\chi\right)\right. \\
\left.+c_{12}^{-} \sin \left(Q_{m} \theta+\chi\right)\right)
\end{array}
$$

where $\chi$ is the phase between the AC dipole and the location $s$, and

$$
\bar{C}=\left(\begin{array}{cc}
\frac{1}{\sqrt{\beta_{u}}} & 0 \\
\frac{\alpha_{u}}{\beta_{u}} & \sqrt{\beta_{u}}
\end{array}\right) C\left(\begin{array}{cc}
\sqrt{\beta_{u}} & 0 \\
-\frac{\alpha_{u}}{\beta_{u}} & \frac{1}{\sqrt{\beta_{u}}}
\end{array}\right)
$$

Hence,

$$
\sqrt{\frac{\beta_{y}}{\beta_{x}}} \frac{1}{\gamma} \sqrt{c_{22}^{-2}+c_{12}^{-2}}=\frac{y_{a m p}}{x_{a m p}}
$$

Similarly, the ratio of the horizontal coherent excitation amplitude to the vertical coherent excitation amplitude is

$$
\sqrt{\frac{\beta_{x}}{\beta_{y}}} \frac{1}{\gamma} \sqrt{c_{11}^{-2}+c_{12}^{-2}}=\frac{x_{a m p}}{y_{a m p}}
$$

when the beam is adiabatically excited by a vertical AC dipole nearby the vertical tune.

\section{EXPERIMENT DATA}

The experiment was carried out in the RHIC blue ring with deuteron beam at injection energy. The linear coupling was corrected to $\delta Q_{\text {min }} \sim 0.001$ at injection for the normal operation using three RHIC skew quadrupole families distributed about $120^{\circ}$ apart [5]. During the experiment, the coupling strength was measured with different skew family 1 strengths. The vertical (horizontal) AC dipole was used to adiabatically induce coherent oscillation nearby the vertical tune (horizontal tune). 1024-turn BPM (Beam Position Monitor) data in both planes were acquired at all the available RHIC BPMs in the arc areas. The amplitude of the coherent oscillation was calculated by fitting the turn by turn betatron oscillation data with a sinusoidal waveform [4].

Fig. 1 is a typical set of data of the measured coherent amplitudes in the two transverse planes due to a vertical ac dipole excitation. Due to the $\beta$-function variations along the arc, the coherent amplitude also varies with the $\beta$-function and the solid line is the average of the measured amplitude within one arc area.

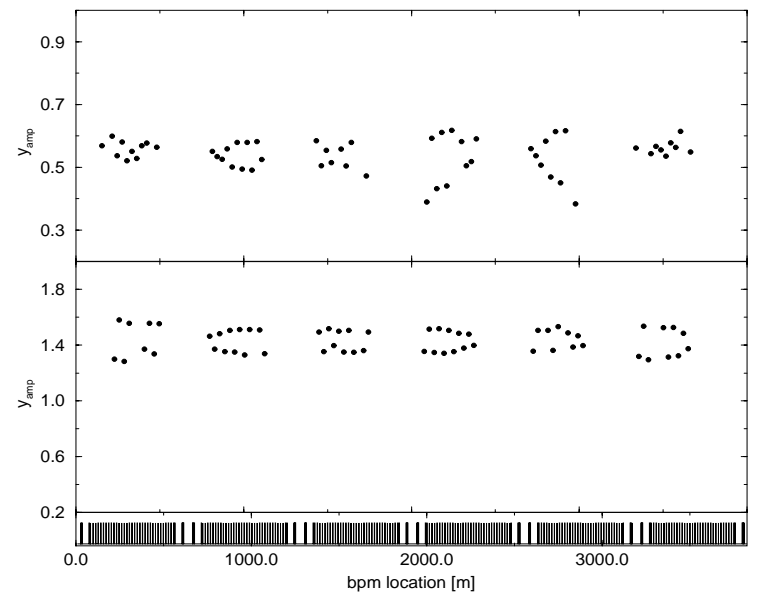

Figure 1: The plot at the bottom is the RHIC lattice. The top plot shows the measured amplitude of the horizontal coherent excitation when the coherent excitation was excited by a vertical AC dipole with skew quad family 1 trim value set to 0.005 . The amplitude of the corresponding vertical coherent excitation amplitude at each BPM is shown in the middle plot.

Fig. 2 shows the measured coupling strength. The bottom plot is the measured $\sqrt{c_{11}^{-}+c_{12}^{2}}$ with the vertical AC dipole and the top plot shows the $\sqrt{{\overline{c_{22}}}^{2}+c_{12}^{-}}$with the horizontal ac dipole. Both measurements consistently show a linear dependence on the skew quadrupole family strength. Both data also show that the minimum of the measured coupling strength occurs at zero trim strength in skew quadrupole family 1 . This is consistent with the fact that the coupling was well compensated at injection during normal operations. 


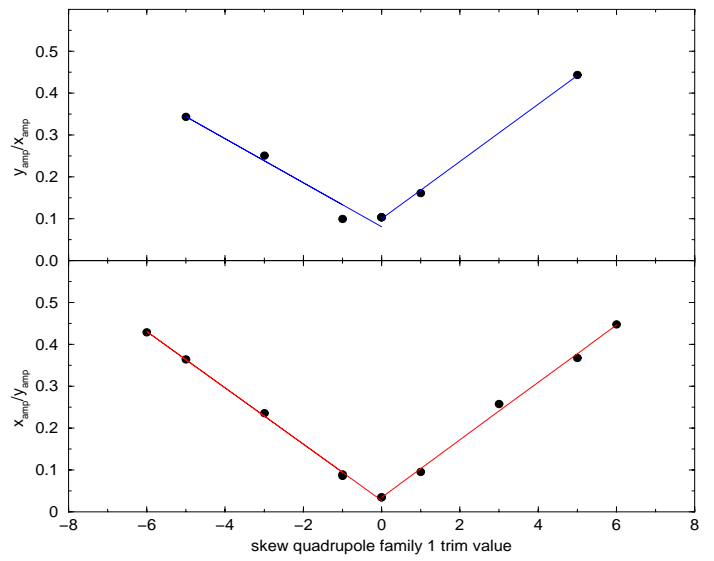

Figure 2: The top plot is the measured $\sqrt{c_{22}^{-}+c_{12}^{-}}$with the horizontal AC dipole as a function of the trim value of skew quadrupole family 1 . And the bottom plot shows the measured $\sqrt{c_{11}^{-}+c_{12}^{2}}$ with the vertical AC dipole with different skew quadrupole family 1 trim value settings.

\section{CONCLUSION}

The method of using driven betatron oscillations to measure the linear coupling resonance was tested during the RHIC FY03 dAu run. It demonstrated that the amount of the coupling can be characterized by the ratio of the amplitude of the horizontal (vertical) oscillation driven by an vertical AC dipole and the amplitude of the corresponding coherent excitation amplitude in the vertical (horizontal) plane. Since this technique does not require changes of the machine working point and is parasitic to the normal machine operation, it can also be used to measure the linear coupling along the RHIC acceleration ramp.

\section{ACKNOWLEDGEMENT}

The authors would like to thank Dr. W. Fischer for the fruitful discussions. The authors would also like to L. Hoff, V. P, J. P and B. Oerter on the constructing the RHIC AC dipole control hardware and software. The authors would also like to thank P. Oddo, J. Delong, T. Russo, D. Lehn, T. Curcio and other engineers for their help.

\section{REFERENCES}

[1] D. A. Edwards, L. C. Teng, Parameterization of Linear Coupled Motion in Periodic Systems, IEEE Trans. Nucl. Sci. 20, 3 (1973).

[2] D. Sagan et al., Betatron phase and coupling measurements at the Cornell Electron/Position Storage Ring Physical Review ST-AB, 3 (2000)

[3] M. Bai et al., Overcoming Intrinsic Spin Resonances with an rf Dipole, Physical Review Letters 80, 4673(1998).

[4] M. Bai et al., Measure Betatron Functions and Phase Advances in RHIC with AC Dipole, Proceedings of the 2003 Particle Accelerator Conference(May 2003)
[5] F. Pilat et al., Coupling measurements and correction at RHIC, Proceedings of the 2002 European Particle Accelerator Conference(June 2002) 